\title{
NUCLEAR MAGNETIC RESONANCE TOMOGRAPHY WITH A TOROID CAVITY DETECTOR
}

\author{
Klaus Woelk, Jerome W. Rathke, and Robert J. Klingler \\ Chemical Technology Division, Argonne National Laboratory
}

A new type of nuclear magnetic resonance (NMR) tomography has been developed at Argonne National Laboratory. The method uses the strong radio frequency field gradient within a cylindrical toroid cavity to provide high-resolution NMR spectral information while simultaneously resolving distances on the micron scale. The toroid cavity imaging technique differs from conventional magnetic resonance imaging (MRI) in that NMR structural information is not lost during signal processing. The new technique could find a wide range of applications in the characterization of surface layers and in the production of advanced materials. Potential areas of application include in situ monitoring of growth sites during ceramic formation processes, analysis of the oxygen annealing step for wires coated with high-temperature superconducting films, and investigation of the reaction chemistry as a function of distance within the diffusion layer for electrochemical processes. The toroid cavity imager was invented by Drs. Klaus Woelk, Jerome Rathke, and Robert Klingler with support from the Division of Chemical Sciences.

\section{DISCLAIMER}

This report was prepared as an account of work sponsored by an agency of the United States Government. Neither the United States Government nor any agency thereof, nor any of their employees, makes any warranty, express or implied, or assumes any legal liability or responsibility for the accuracy, completeness, or usefulness of any information, apparatus, product, or process disclosed, or represents that its use would not infringe privately owned rights. Reference herein to any specific commercial product, process, or service by trade name, trademark, manufacturer, or otherwise does not necessarily constitute or imply its endorsement, recommendation, or favoring by the United States Government or any agency thereof. The views and opinions of authors expressed herein do not necessarily state or reflect those of the United States Government or any agency thereof. 


\section{DISCLAIMER}

\section{Portions of this document may be illegible in electronic image products. Images are produced from the best available original document.}




\section{NUCLEAR MAGNETIC RESONANCE TOMOGRAPHY WITH A TOROID CAVITY DETECTOR \\ Klaus Woelk, Jerome W. Rathke, and Robert J. Klingler \\ Chemical Technology Division, Argonne National Laboratory \\ 9700 South Cass Avenue, Argonne, IL 60439}

A new type of nuclear magnetic resonance (NMR) tomography has been developed at Argonne National Laboratory that allows the measurement of high-resolution NMR spectral information while simultaneously resolving distances on the micron scale. The new tomography method uses a toroid cavity detector for rotating frame spectroscopic imaging. The toroid cavity resonator produces a well-defined gradient with respect to distance in the alternating magnetic field, $B_{1}$. As a result of this transmitter/detector coil modification, the amount of energy that is absorbed/transmitted varies with the location of the sample within the NMR detector because the efficiency of the energy transfer process is proportional to $B_{1}$. Furthermore, multiple distances within the NMR active sample can be resolved by varying the transmitter pulse length. Significantly, the toroid cavity resonator is unique in the accuracy of the $B_{1}$ field gradient. This precise $B_{1}$ field gradient provides distance measurements on the micron scale for materials that are close to the central conductor of the toroid cavity resonator. A second major advantage of the toroid cavity imaging (TCI) approach to magnetic resonance imaging (MRI) is that the chemical shift information is not destroyed because the measurement is conducted within a homogeneous static magnet field, $B_{0}$. In contrast, the traditional approach to MRI proceeds by converting the chemical shift information to a measure of distance with a gradient in $B_{0}$. A related advantage of the new approach to NMR imaging is that the TCI probe may be inserted as a simple replacement for the standard detector in conventional Fourier transform NMR spectrometers. The magnet shim system does not need to be altered to produce a gradient in $B_{0}$.

The toroid cavity resonator that was used for these studies is depicted in the first figure. This detector design has several advantages. It provides a rugged reaction chamber that is readily machined from a variety of alloys. In addition, the alternating magnetic field is highly confined within the cavity resonator. This minimizes sensitivity losses that occur through magnetic coupling with the metallic walls of the probe housing. Most importantly, the cavity resonator produces a well-defined magnetic field gradient, which is predicted to vary with the inverse of the radial displacement from the center of the cavity. Thus, the alternating magnetic field is 
strongest near the central conductor of the cavity and drops off toward the outer walls. This field dependence was confirmed experimentally by extending the solution of the Bloch equations to include the inhomogeneous $B_{1}$ field of the toroid cavity. The resultant NMR intensity $\left(I / I_{0}\right)$ is predicted to depend on the transmitter pulse length, $t$, according to eq 1 ,

$$
I / I_{0}=2 \pi h \int \sin \left(\frac{\gamma A t}{r}\right) \mathrm{d} r
$$

where $\gamma$ is the gyromagnetic ratio, $h$ is the height of the toroid, $r$ is the radial distance from the center of the cavity, and $A$ is the proportionality constant defining the magnetic field in eq 2 .

$$
B_{1}=\frac{A}{r}
$$

The second figure illustrates the agreement between the experimental intensity distribution in the ${ }^{1} \mathrm{H}$ NMR spectra for a chloroform sample with the theoretical prediction of eq 1 . These results demonstrate that the toroid cavity resonator has an accurately-defined $B_{1}$ field gradient. Furthermore, it is this $B_{1}$ field gradient which provides the basis for high-resolution distance measurements in the TCI approach to NMR imaging.

The TCI technique simultaneously provides high-resolution chemical shift and distance resolution, as demonstrated by the results in the third figure. This experiment was conducted with five small capillaries that were filled with different solvents and equally spaced within the toroid cavity resonator. Both the diameter and radial distance from the center of the cavity were accurately reproduced for each of the five capillaries. Significantly, the chemical shift information was retained. Thus, in a single experiment, the TCI technique can chemically distinguish between water and acetone, in addition to identifying the location of these samples along the distance axis. The chemical shift resolution of the TCI technique should be equivalent to that of a normal NMR spectrum. Alternatively, the ultimate resolution with respect to distance for the toroid cavity detector is under study. The data in the fourth figure demonstrate the distance resolution by ${ }^{19} \mathrm{~F}$ NMR spectroscopy of two Teflon layers that were placed around the central conductor of the toroid cavity resonator. The measured thickness of the Teflon layers, as well as their $20 \mu \mathrm{m}$ separation, is accurately reproduced by the imaging data. 
The toroid cavity resonator has a unique advantage in that both the NMR sensitivity and the distance resolution should increase for materials that are close to the central conductor due to the inverse radial dependence for the $B_{1}$ field in eq 2 . Thus, the TCI technique is envisioned to be particularly powerful in the characterization of surface layers and in materials analysis. Potential areas of application for the TCI approach to NMR imaging include the in situ NMR monitoring of growth sites during ceramic formation processes. For example, it should be possible to monitor both the gas phase and the active surface sites during chemical vapor deposition by heating the central conductor of the toroid cavity resonator. In addition, the toroid cavity detector is well suited for use in high-pressure metal probe housings. Thus, a supercritical fluid at high pressure can be used as the solvent medium to accelerate the diffusion of viscous preceramic polymer reactants into permeable composite materials. Alternatively, if a wire coated with a high-temperature superconducting ceramic is heated, it should be possible to follow the reaction chemistry for the key oxygen annealing process. In addition, the equilibrium compositions of the various phases in the superconducting material may be probed as a function of penetration depth and oxygen partial pressure by the TCI method. Another potential application is to use the central conductor of the toroid cavity resonator as a electrode in an electrochemical cell. A nearly constant electrolysis current will not impede the high-frequency NMR measurement, and the $300 \mathrm{MHz}$ NMR transmitter pulse is an alternating signal that is well above the time domain of most important electrocatalytic processes. As a result, the toroid cavity resonator may lead to an in situ NMR technique for the investigation of the reaction chemistry as a function of distance within the diffusion layer of electrochemical processes. 
Outer Diameter of

Cavity: $16.3 \mathrm{~mm}$
Volume inside

Cavity: $2.57 \mathrm{ml}$

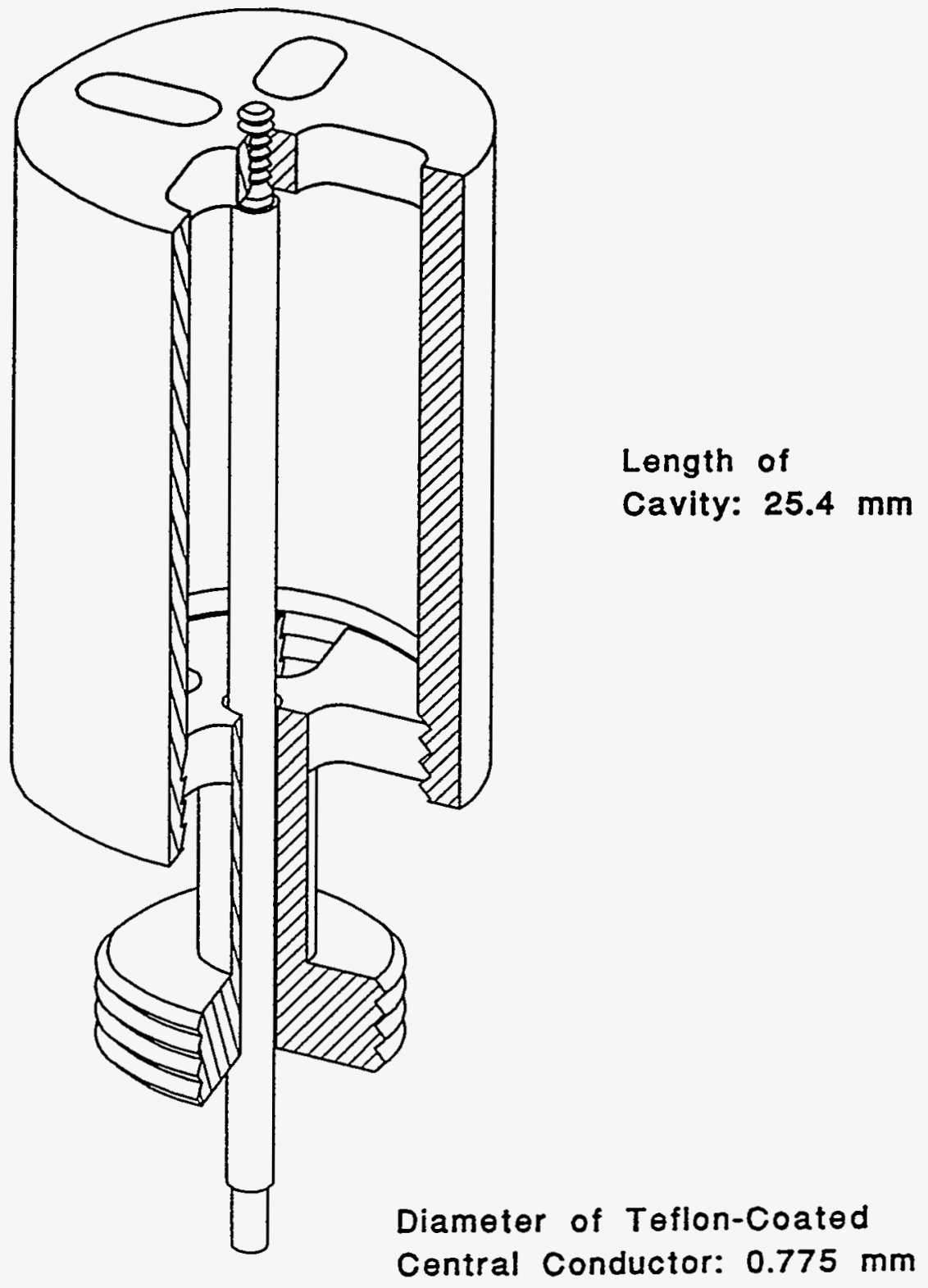


NMR Signal Intensity as a Function of Pulse Width, Obtained from a Homogeneously Filled Toroid Cavity

- Experimental data

- Calculated curve

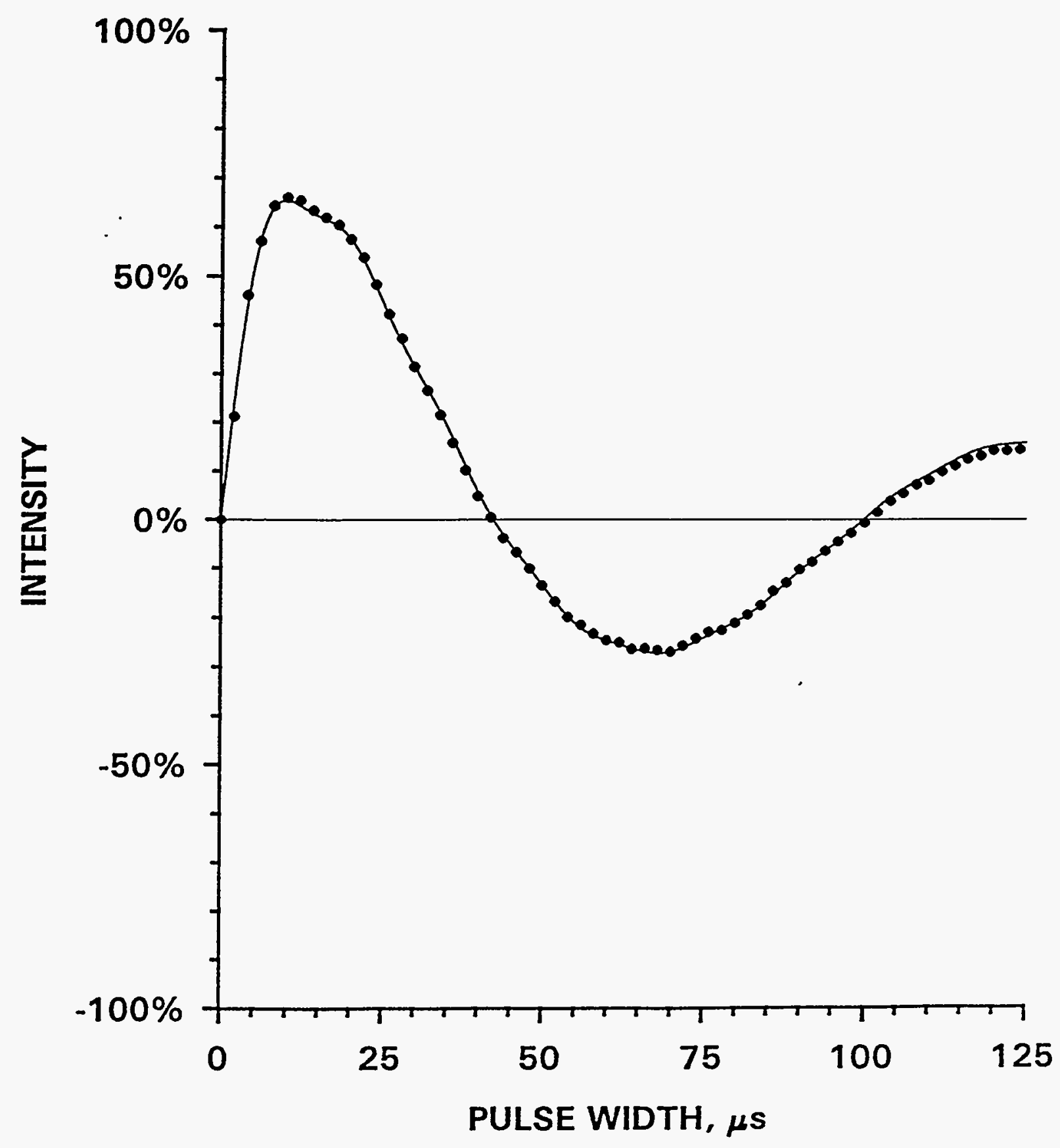




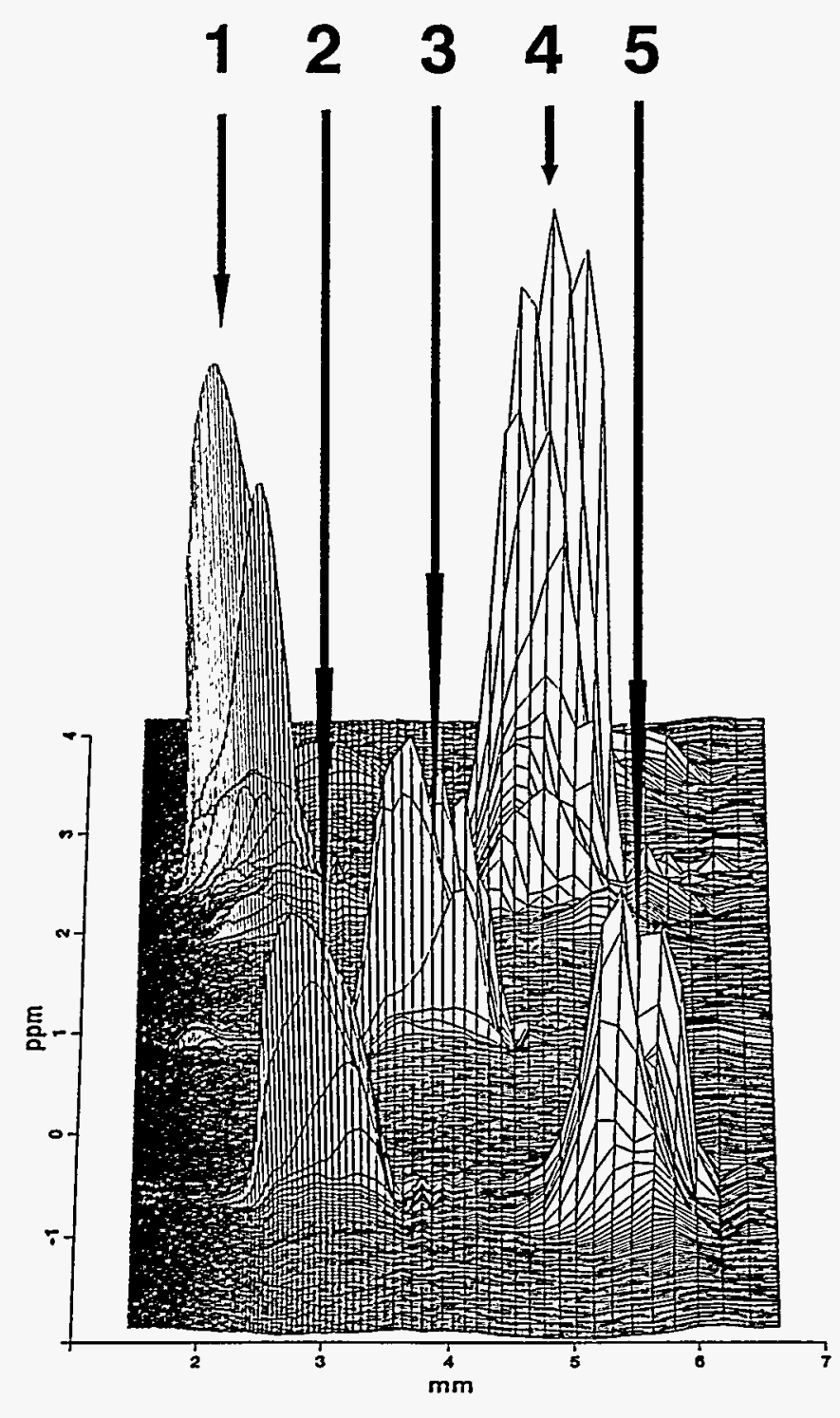

1.) Water

2.) Isopropanol

3.) Acetone

4.) Water

5.) Isopropanol

NMR TOMOGRAPHY in a TOROID CAVITY

Demonstration of the simultaneous spacial and chemical shift resolution of five $1 \mathrm{~mm}$ capillaries each spaced $0.8 \mathrm{~mm}$ apart radially from the cavity center

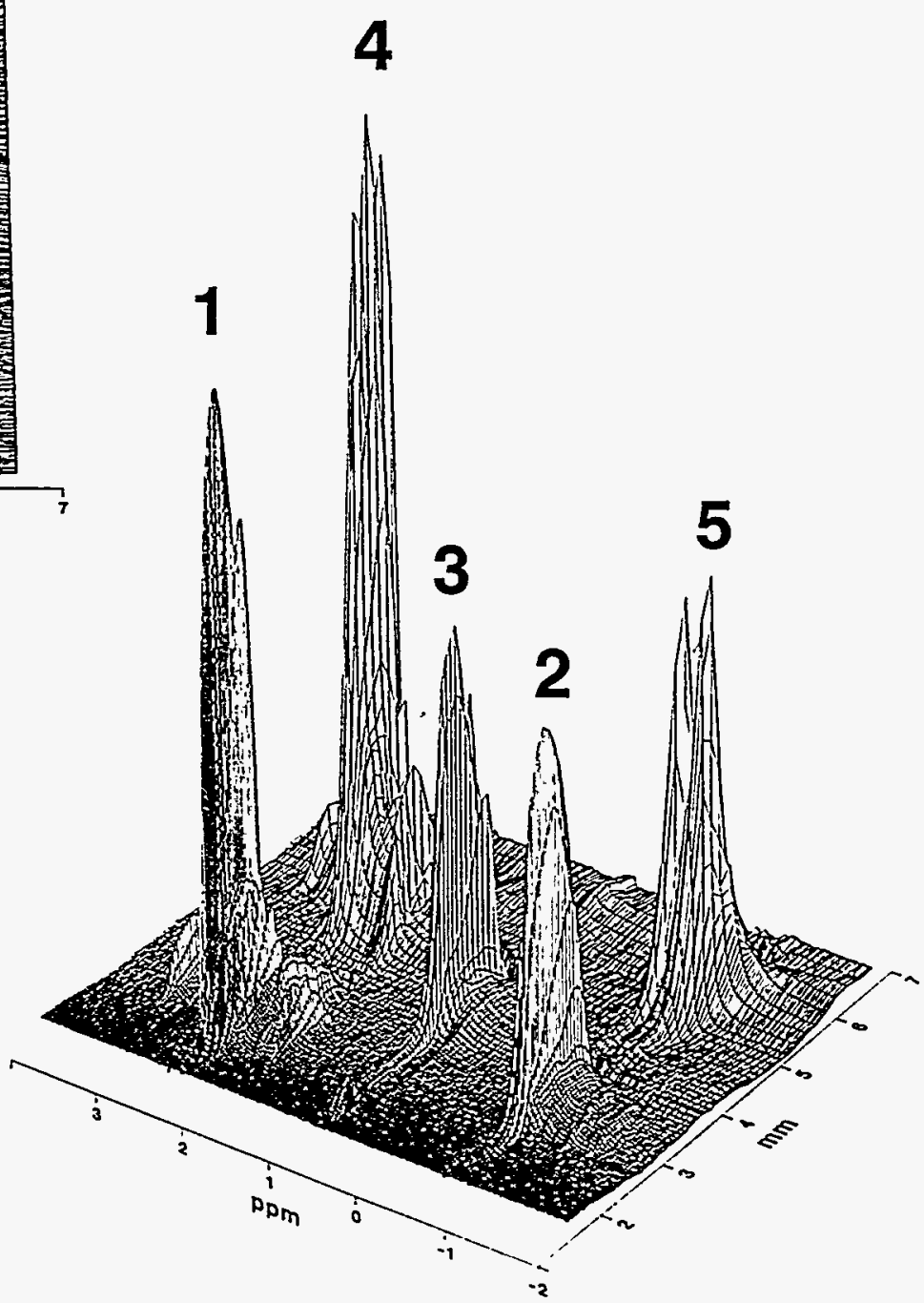


${ }^{19}$ F-NMR Signal of Teflon vs. Radius of Toroid Cavity

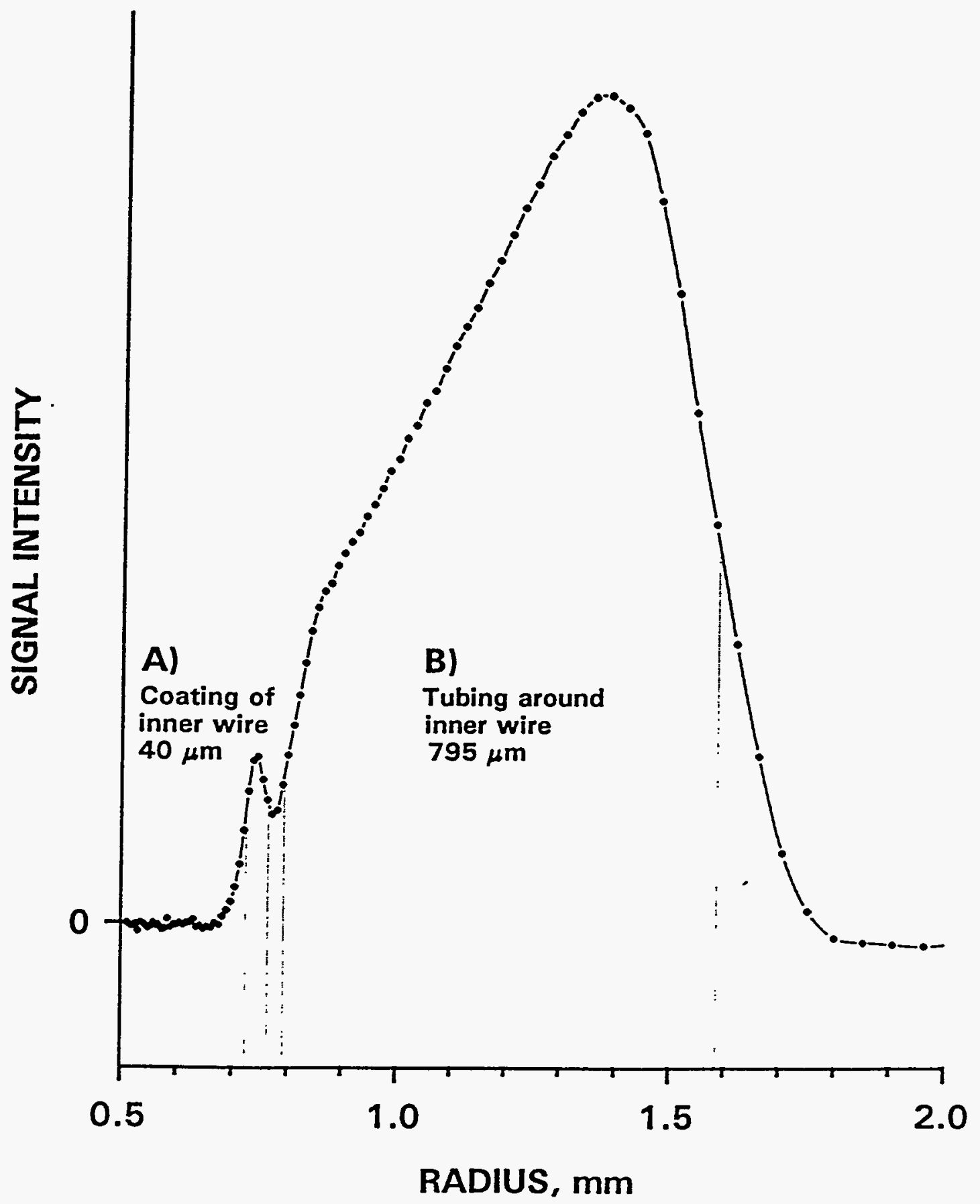

Ratio $A / B=0.051$ (manufacturer's specifications)

$A / B=0.048$ (experiment)

Resolution $<20 \mu \mathrm{m}$ 